\title{
Mesogene-Polymer Backbone Coupling in Side-Chain Polymer Liquid Crystals, Studied by High Magnetic-Field-Induced Alignment
}

\author{
M. I. Boamfă ${ }^{1, *}$ K. Viertler, ${ }^{2}$ A. Wewerka, ${ }^{2}$ F. Stelzer, ${ }^{2}$ P. C. M. Christianen, ${ }^{1}$ and J. C. Maan ${ }^{1}$ \\ ${ }^{1}$ Research Institute for Materials, High Field Magnet Laboratory, University of Nijmegen, Toernoiveld 1, 6525 ED, The Netherlands \\ ${ }^{2}$ Institute for Chemistry \& Technology of Organic Materials, Graz University of Technology, Stremayrgasse 16/1, \\ A-8010 Graz, Austria
}

(Received 29 May 2002; published 14 January 2003)

\begin{abstract}
We show that cooling side chain polymer liquid crystals in a magnetic field, from the isotropic to nematic and subsequently to the glass phase, results in a macroscopically ordered, transparent, and strongly birefringent material. The aligned samples retain their properties after the field is removed and can be dealigned only by heating them to the isotropic phase. To induce alignment, a threshold field $B_{\text {th }}$ is necessary, which strongly depends on the chemical structure details. $B_{\text {th }}$ reflects the strength of mesogene-polymer backbone coupling, and we will show that this interaction is responsible for the stability of the induced alignment at zero field.
\end{abstract}

DOI: $10.1103 /$ PhysRevLett.90.025501

PACS numbers: 61.30.Gd, 61.41.+e, 64.70.Md

Side chain polymer liquid crystals (SCPLCs) [1] are composed of rigid mesogenic units attached to a polymeric backbone chain via a flexible spacer (Fig. 1), with enough freedom for the mesogenes to form liquid crystalline mesophases. These molecules combine the self-organizing properties of liquid crystals with the structural advantages of polymers and are interesting for applications [2] like optically nonlinear devices [3], displays [4,5], and optical data storage [6]. Their current limiting factor is the lack of a reliable method to control the SCPLCs molecular alignment at a macroscopic scale. A large number of techniques are used for alignment, such as surface fields [7], electric fields [8], mechanical fields [9], flow fields [10], or polarized ultraviolet light [11], but only with relative success.

Here we show that perfectly oriented SCPLC films are obtained by cooling them in a magnetic field from the isotropic to nematic phase. Since field alignment of the SCPLCs at a fixed temperature is found to be impossible, we conclude that alignment occurs only in the biphasic region at the isotropic nematic phase boundary [12]. Here small liquid crystalline nuclei are formed and undergo orientation in the small temperature window, where the mesogene-backbone interaction is weaker than the magnetic field effect. Upon cooling, the system evolves into a monodomain, strongly birefringent sample, which retains its properties after the field is switched off. This aligned material is technologically interesting because of its strong birefringence and forms an ideal target for further scientific studies like x-ray or neutron scattering experiments. In comparison with other alignment techniques [7-11], magnetic alignment has several advantages, since the field exerts a well-defined, contact free, torque force on all molecules, and can therefore be used for thin films as well as bulk samples.

Low molecular weight liquid crystals can be perfectly aligned in magnetic fields [13], but for liquid crystalline polymers the alignment process is less effective or time consuming [14-19] because the rotational freedom of the mesogenes is hindered by the polymer backbones. We observe this hindrance and determine its strength, because the degree of alignment of different SCPLCs, each
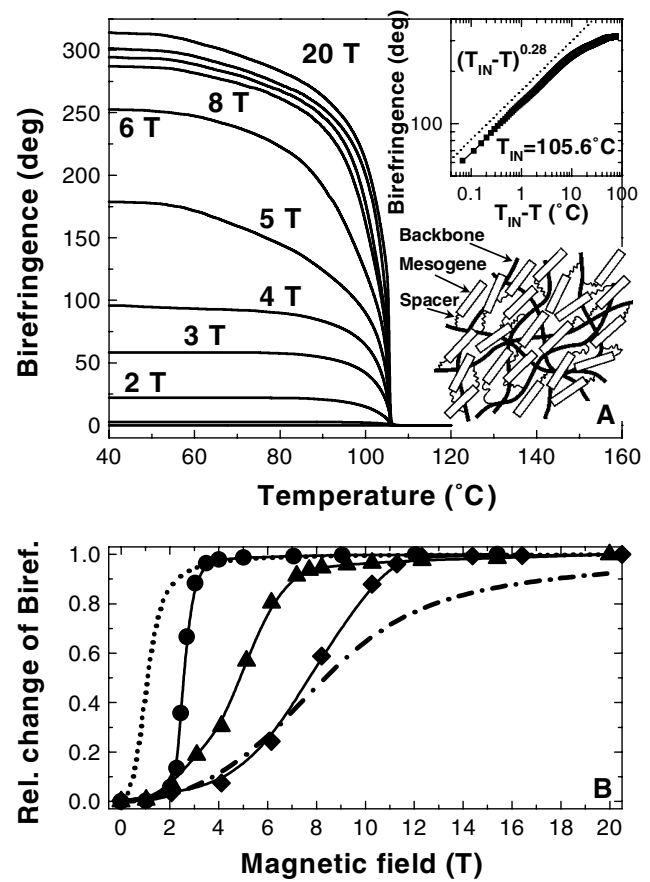

FIG. 1. Birefringence of SCPLCs cooled in different magnetic fields; (a) A $10 \mu \mathrm{m}$ thick $C 5$ sample cooled from isotropic to glass phase; upper inset: the birefringence of the same film at 12T; lower inset: schematic representation of the SCPLCs structure. (b) The saturation values of the birefringence in the glass phase of different SCPLCs: $C 5, n=20$ (triangles); $C 5, n=30$ (diamonds); and $B 4, n=30$ (circles), together with numerical calculations that neglect the backbone-mesogene coupling (dotted and dash-dotted lines). 
containing the same mesogenic units, is found to depend strongly on the chemical properties of their backbone and spacer. A proper understanding of the mesogenebackbone interaction is one of the key issues in order to develop the SCPLCs into useful materials. Experimentally the determination of this interaction is an open question [20], and only recently neutron scattering experiments $[10,21,22]$ have shown that the polymeric backbone configuration can indeed be correlated to the local mesogene director.

We used two types of thermotropic nematic SCPLCs, based on a polynorbornene or a polyacrylate-derivative backbone, each with cyanobiphenyl mesogenic groups. The molecular structures are shown in Table I, together with their measured phase transition temperatures. The polynorbornene based SCPLC has a relatively stiff backbone due to the double bonds that connect the cyclopentylene units, and is synthesized by ring opening metathesis polymerization, with a low polydispersity $P D I \sim$ 1.2 [23]. Two different spacers were used consisting of either three or five methylene units, configurations which we denote as $C 3$ and $C 5$, respectively. The polymerization degree of $C 5$ was varied from $n=20$ to 40 . The backbone of the polyacrylate derivative (B4), synthesized by free radical polymerization $(P D I \sim 2)$, is more flexible than the polynorbornene. In this case a spacer length of four methylene units was used, with the polymerization degrees being $n=20$ and 30 .

Film samples (thickness 10-200 $\mu \mathrm{m}$ ) were prepared on a glass substrate by spin coating from solution and subsequent solvent removal by thermal annealing. The

TABLE I. Molecular structures and material parameters of the samples.

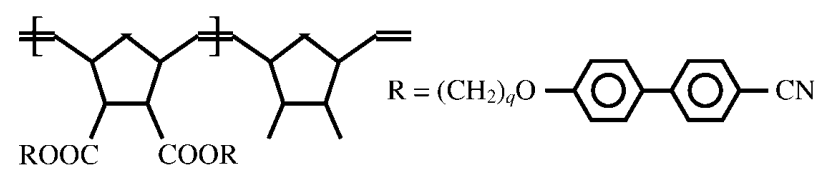

Poly[( \pm )-exo,endo-bis $\{q$-[(4'-cyanobiphenyl-4-yl)oxy]pentyl $\}$ norborn-5-ene-2,3-dicarboxylate], with $q=3$ (C3) or $q=5$ (C5)

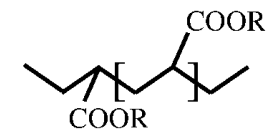

$$
\mathrm{R}=\left(\mathrm{CH}_{2}\right)_{4} \mathrm{O} \mathrm{O}-\mathrm{O}-\mathrm{CN}
$$

Poly- $\left\{4-\left[\left(4^{\prime}\right.\right.\right.$-cyanobiphenyl-4-yl)oxy]butyl $\}$ acrylate (B4)

\begin{tabular}{lcccccc}
\hline & & $K_{m / b}$ & Align. preserved & \multicolumn{3}{c}{ Trans. Temp } \\
Sample & $B_{\mathrm{th}}(\mathrm{T})$ & $(\mathrm{Nm} / \mathrm{molec})$ & upon cooling & $\beta$ & $T_{\mathrm{G}}$ & $T_{\mathrm{IN}}\left({ }^{\circ} \mathrm{C}\right)$ \\
\hline$C 5, n=20$ & 6 & $3.6 \cdot 10^{-26}$ & Yes & 0.28 & 43 & 105.6 \\
$C 5, n=30$ & 10 & $10 \cdot 10^{-26}$ & Yes & 0.28 & 43 & 109.2 \\
$C 5, n=40$ & 19 & $36 \cdot 10^{-26}$ & No & 0.28 & 45 & 112.5 \\
$C 3, n=20$ & $>20$ & $\ldots$ & No ind. effect & 0.10 & 57 & 92.6 \\
$B 4, n=20$ & 2 & $0.4 \cdot 10^{-26}$ & Yes & 0.40 & 43 & 94.0 \\
$B 4, n=30$ & 3.5 & $1.2 \cdot 10^{-26}$ & Yes & 0.40 & 43 & 96.7 \\
\hline
\end{tabular}

samples were placed in a temperature-controlled chamber inside the bore of a $20 T$ resistive Bitter-magnet. All experiments were done at a cooling rate of $10^{\circ} \mathrm{C} / \mathrm{min}$, which was sufficiently slow for the samples to be always in equilibrium. The SCPLCs order parameter $[15,16]$ was probed in situ via linear birefringence measurements [24], using a polarization modulated $\mathrm{HeNe}$ laser $(543.5 \mathrm{~nm})$.

Figure 1(a) shows the measured birefringence during cooling down of a $10 \mu \mathrm{m}$ thick $C 5, n=20$ sample, from $120^{\circ} \mathrm{C}$, through the isotropic to nematic transition, $T_{\mathrm{IN}}$, and subsequently until the glass phase $T_{G}$ is reached, for different magnetic fields. No induced birefringence is observed either in the isotropic phase for all fields or in zero magnetic field in the nematic phase, although in the latter case local order exists inside randomly oriented micron sized domains [see Fig. 2(a)]. The strong variations of the local refractive index leads to scattering and results in an overall opaque appearance [see Fig. 2(b)]. In finite fields birefringence appears around $T_{\mathrm{IN}}$, which increases upon further cooling and saturates at $T_{G}$ [Fig. 1(a)]. With increasing fields the saturation value increases, but the onset of the birefringence always occurs around $T_{\mathrm{IN}}$. The inset of Fig. 1(a) shows that around $T_{\mathrm{IN}}$ the induced birefringence follows a $\left(T_{\mathrm{IN}}-T\right)^{\beta}$ power law [13]. The critical exponent $\beta$ has different values for the different SCPLCs (see Table I), but within a family its value does not depend on the polymerization degree or the magnetic field strength. All samples, except the $C 5$, $n=40$ [25], remain aligned after removal of the field and show no further change, even after several months. The oriented material exhibits uniaxial monodomain alignment [Fig. 2(c)], full transparency [Fig. 2(d)], and large birefringence $(\Delta n=0.1)$, which corresponds to a director order parameter $[15,16] Q_{D}=0.999 \pm 0.001$.

The saturation value of the induced birefringence, trapped in the glass phase, as a function of the applied magnetic field for three different samples is plotted in Fig. 1(b). All samples show a gradual rise in the birefringence at low fields, followed by a rapid increase and saturation at high field.

All experimental evidence shows that alignment merely occurs in a narrow temperature window around $T_{\mathrm{IN}}$. For instance, heating of a prealigned sample at zero field leads to a very similar birefringence curve as the original one measured during cooling in a field (Fig. 3). Reversing such a temperature sweep of a prealigned sample before reaching $T_{\mathrm{IN}}$ just restores the initial aligned state. Finally, attempts to magnetically align a SCPLC film at a fixed temperature always failed. Although all data reported here are on films, we have obtained the same results with bulk material in $20 \times$ $10 \times 2 \mathrm{~mm}^{3}$ cuvettes.

In order to explain these observations the alignment mechanism should be considered in more detail. Because of the anisotropy of its diamagnetic susceptibility $\Delta \chi$, a 

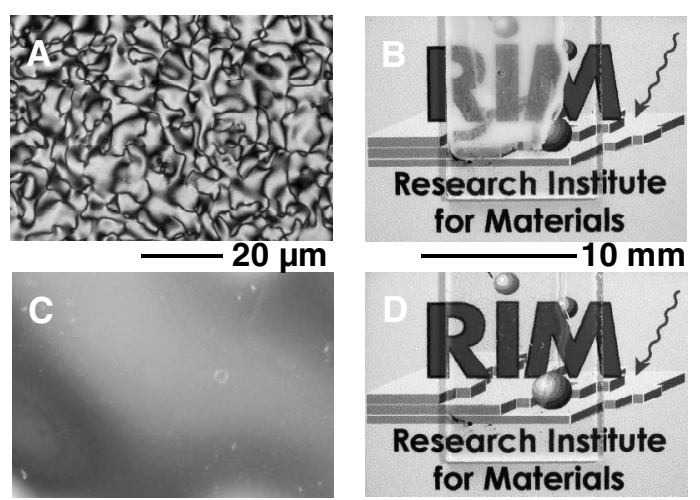

FIG. 2. Optical properties of magnetically aligned SCPLC films. (a) Crossed polarized microscope image, of an unprocessed $10 \mu \mathrm{m}$ thick $B 4, n=20$ film, micrometer sized domains. (b) Corresponding photograph: the domains cause light scattering. (c) Crossed polarized microscope image of a $10 \mu \mathrm{m}$ thick $B 4, n=20$ film, aligned in a $5 T$ field, macroscopic monodomain; the color variations are due to small thickness variations. (d) Corresponding photograph: the aligned sample is fully transparent.

molecule tends to orient relative to an applied magnetic field $(B)$ such that its energy is minimized. For single molecules, the reduction in magnetic energy is small compared to the thermal energy $\left(\Delta \chi B^{2} \ll k T\right)$. Therefore, SCPLCs within the isotropic phase exhibit no birefringence in a magnetic field. However, for collective molecular behavior, like in liquid crystalline mesophases, the difference in magnetic energy, added up for all molecules within a correlation volume $\left(N_{\xi}\right)$, can become large enough $\left(N_{\xi} \Delta \chi B^{2}>k T\right)$ to induce magnetic alignment [13,24]. Quantitatively, for a biphenyl group $\left(\Delta \chi=120 \times 10^{-6} \mathrm{~cm}^{3} / \mathrm{mol}\right.$ [24]), $10^{4}$ molecules are sufficient to achieve alignment at room temperature and field strengths of $10 \mathrm{~T}$.

During cooling, nematic nuclei are formed around $T_{\mathrm{IN}}$ [12] and rapidly grow in size with decreasing temperature (biphasic region, see Fig. 3). When these nuclei are sufficiently large $\left(N_{\xi}>10^{4}\right)$, they are aligned by the field. The actual alignment occurs in a narrow temperature window at the beginning of the isotropic nematic transition, characterized by the sharp onset of the birefringence at $T_{\text {IN }}$ [Fig. 1(a)]. The subsequent increase of the birefringence farther away from the biphasic region, characterized by the critical parameter $\beta$, is caused by the usual, purely temperature driven, alignment of the mesogenes with respect to the prealigned director [13] and is field independent. That is, the local order parameter $Q_{N}$, describing the order within a correlation volume, is unaffected by the magnetic field, which affects only the director order parameter $Q_{D}$, leading to a high total order parameter $Q(B, T)=Q_{N}(T) Q_{D}(B)[15,16]$, as measured in the birefringence.

Calculations of the magnetic alignment of bare cyanobiphenyl units, neglecting the coupling to the polymer

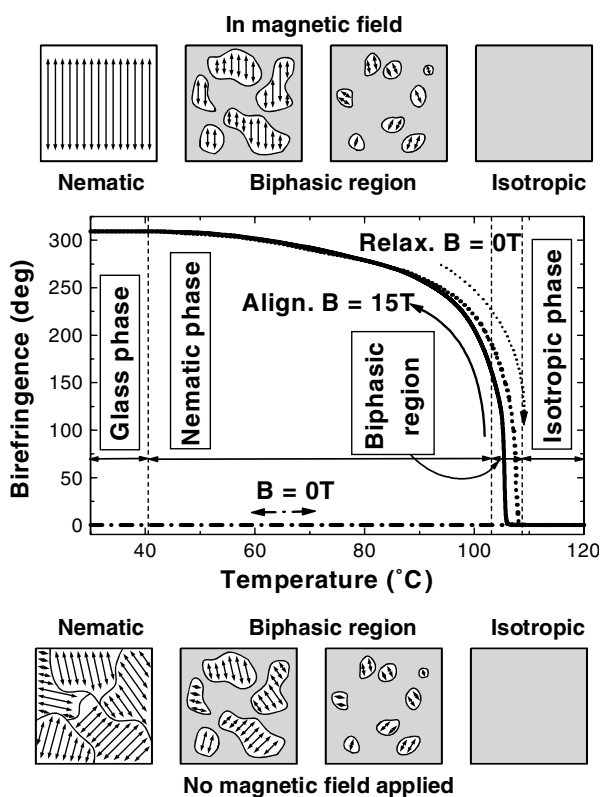

FIG. 3. Magnetic field induced alignment of SCPLCs. The birefringence of a $C 5, n=10$ film of $20 \mu \mathrm{m}$ thickness cooled in $15 \mathrm{~T}$ (solid curve) and subsequently heated in zero field (dashed curve). At zero field no birefringence is measured (dash-dotted curve) due to random director orientation in different domains (bottom panel). In a magnetic field the directors of the liquid crystalline nuclei point in the same direction, resulting in the development of a macroscopic monodomain (top panel). The top and bottom panel are based on optical microscope observations.

backbone, are shown in Fig. 1(b). The director order parameter is governed only by the $N_{\xi} Q_{N} \Delta \chi B^{2} / k T$ ratio. $Q_{D}$ describes a Langevin type of function, showing no alignment $\left(Q_{D}=0\right)$ at low fields and saturation $\left(Q_{D}=1\right)$ at high fields [13]. The curves reasonably describe the low (high) field results, but it is impossible to describe both low and high field behavior simultaneously with a single fit parameter $N_{\xi} Q_{N} \Delta \chi / k T$. In particular, the measured magnetic alignment saturates rather abruptly even if at low fields no effect is seen, pointing to a certain threshold field $B_{\text {th }}$, which must be overcome to induce alignment.

The backbone does not contribute actively to the mesophase and is not directly influenced by the magnetic field, but its presence hinders the mesogene freedom. Because the free volume per molecule in the nematic phase does not allow a free molecular rotation, any change of orientation of a mesogene needs to be accompanied by a local rearrangement of the surrounding polymer chains. The thermal hysteresis of the IN transition (Fig. 3) and the existence of sharp domain walls [Fig. 2(a)] indicate consistently the existence of entanglements and conformational barriers that impend the mesogene mobility [24]. We interpret this behavior as consistent with the existence of a threshold field $B_{\mathrm{th}}$ and indicative of mesogene-backbone interaction. The mesogene-backbone coupling accounts for all the backbone obstructions to the 
mesogene mobility and can be described as an "effective torque" $K_{m / b}$ acting at molecular level [26]. $K_{m / b}$ opposes the magnetic torque and acts as a barrier, leading to a threshold field $B_{\text {th }}$, above which magnetic field alignment can occur. $B_{\text {th }}$ will therefore reflect the balance between the coupling torque and the magnetic torque, $B_{\mathrm{th}}=\sqrt{3 K_{m / b} / 2 \Delta \chi Q_{N}}$ [27]. By defining $B_{\mathrm{th}}$ as the field needed to produce $50 \%$ of the saturated birefringence, we calculate $K_{m / b}$ at $T_{\mathrm{IN}}$ for all samples measured. The resulting values, given in Table I, show that for the same spacer length (see the $C 5$ compounds) the coupling increases with increasing polymerization degree $(n=20$, 30 , to 40) as may be expected from the increasing entanglement. For the same $n=20$ but changing the spacer length (compare $C 5$ to $C 3$ ), an increasing spacer rigidity (C3) weakens the mesophase and effectively prohibits magnetic alignment. Finally, the polyacrylates (B4) exhibit a lower coupling than the polynorbornenes (C5), which is to be expected because their main chain is more flexible, while they also show the same trend of increased coupling with increasing polymerization degree.

The rather high value of the mesogene-backbone coupling (valid around $T_{\mathrm{IN}}$ ) $K_{m / b} \sim 10^{-25} \mathrm{~N} \mathrm{~m} /$ molecule, is responsible for the alignment stability when the field is removed. The energy needed to rotate a typical correlated volume of SCPLC mesogenes in the nematic phase, which contains about $10^{9}$ molecules, is about $10^{4}$ times larger than the available $k T$ energy.

In summary, we have shown that high optical quality, strongly birefringent SCPLC films can be produced, by cooling them from the isotropic to glass phase in a magnetic field, which opens the way to many possible applications for these highly ordered materials. The degree of alignment strongly depends on the chemical nature of the SCPLC compound. SCPLCs with long polymer chains are more difficult to orient compared to those containing shorter ones, shorter spacers prevent alignment, and SCPLCs with flexible chains are easier to orient than more rigid ones. For best results a welldefined degree of polymerization is necessary which has to be optimized for every polymer system. These results are interpreted in terms of an effective torque coupling between the mesogenic units and the surrounding polymer, leading to the quantitative determination of this interaction.

*Corresponding author.

Email address: boamfa@ sci.kun.nl

[1] H. Finkelmann, H. Rigsdorf, and J.H. Wendorff, Macromol. Chem. 179, 273 (1978).

[2] C. S. Hsu, Prog. Polym. Sci. 22, 829 (1997).

[3] J. C. Dubois et al., Liq. Cryst. 14, 197 (1993).
[4] M. Murakami and H. Fujii, Mol. Cryst. Liq. Cryst. 225, 259 (1993).

[5] $\mathrm{P}$ van de Witte et al., Jpn. J. Appl. Phys. Part 1 38, 748 (1999).

[6] M. Sawodny, Makromol. Chem. 46, 217 (1991).

[7] V. N. Raja et al., Liq. Cryst. 20, 41 (1996).

[8] R. B. Findlay and A. H. Windle, Mol. Cryst. Liq. Cryst. 206, 55 (1991).

[9] Y. Zhao, P. Roche, and G. X. Yuan, Macromolecules 29, 4619 (1996).

[10] C. Pujolle-Robic and L. Noirez, Nature (London) 409, 167 (2001).

[11] N. Kawatsuki, T. Yamamoto, and H. Ono, Appl. Phys. Lett. 74, 935 (1999).

[12] The IN transition of SCPLCs is a weak first order transition, it takes place over a wide temperature region, where the nematic mesophase coexist with the isotropic melt. C. Pugh and A. L. Kiste, in Handbook of Liquid Crystals, edited by D. Demus et al. (Wiley-VCH, Weinheim, 1998), Vol. 3, p. 170.

[13] P.G. de Gennes and J. Prost, The Physics of Liquid Crystals (Clarendon, Oxford, 1993), 2nd ed.

[14] W. R. Krigbaum, in Polymer Liquid Crystals, edited by A. Ciferri (Academic, New York, 1982), Chap. 10.

[15] A. M. Donnald and A. H. Windle, Liquid Crystalline Polymers (Cambridge University Press, Cambridge, 1992).

[16] U.W. Gedde and G. Wilberg, in Mechanical and Thermophysical Properties of Polymer Liquid Crystals, edited by W. Brostow (Chapman \& Hall, London, 1998).

[17] Y. Zhao and H. L. Lei, Macromolecules 25, 4043 (1992).

[18] P. Roche and Y. Zhao, Macromolecules 28, 2819 (1995).

[19] K. Akagi and H. Shirakawa, Curr. Trends Polym. Sci. 2, 107 (1997).

[20] R. G. Kirste and H. G. Ohm, Macromol. Chem. Rapid Commun. 6, 179 (1985).

[21] L. Noirez, P. Keller, and J. P. Cotton, Liq. Cryst. 18, 129 (1995).

[22] L. Noirez, M. Ungerank, and F. Stelzer, Macromolecules 34, 7885 (2001).

[23] M. Ungerank, B. Winkler, E. Eder, and F. Stelzer, Macromol. Chem. Phys. 196, 3623 (1995).

[24] G. Maret and K. Dransfeld, in Strong and Ultrastrong Magnetic Fields and Their Applications, edited by F. Gerlach (Springer-Verlag, Berlin, 1985), Chap. 4.

[25] The $C 5, n=40$ sample can be aligned above $19 \mathrm{~T}$, but loses its alignment during cooling down, possibly due to the buildup of thermal stress close to $T_{G}$, primarily important for polymers with relatively long chains.

[26] This quantity is the differential of the energy dissipated to overcome the coupling, for a given mesogene rotation.

[27] Obtained from the torque balance equation of a correlated volume. The rotational viscosity is rigorously considered, but its contribution affects only the time scale of the alignment. This effect seems the rotational analog of the threshold shear stress needed to initiate flow in Bingham plastic materials. R. B. Bird, R. C. Armstrong, and O. Hassager, Dynamics of Polymeric Liquids (Wiley, New York, 1987), 2nd ed. 